\title{
Weiterer Meilenstein auf dem Weg zu ST Reha erreicht
}

\section{Bruno Trezzinia, Beatrix Meyer ${ }^{b}$}

${ }^{a}$ Dr. phil., wissenschaftlicher Mitarbeiter, Abteilung Stationäre Versorgung und Tarife, FMH; beiterin Abteilung Stationäre Versorgung und Tarife, FMH

Die FMH hat zusammen mit den Fachgesellschaften einen wesentlichen Beitrag zur Abbildung der stationären Rehabilitation in der CHOP-Klassifikation geleistet. Als jüngsten Meilenstein hat sie einen Kompromiss mit den Partnern der SwissDRG AG hinsichtlich der personellen und infrastrukturellen Minimalanforderungen zur Leistungserbringung erreicht.

Der Weg zur Entwicklung der Tarifstruktur ST Reha für die stationäre Rehabilitation ist bisher keineswegs geradlinig verlaufen und war zuweilen steinig. 2018 hat sich der Verwaltungsrat der SwissDRG AG definitiv für einen Richtungswechsel entschieden; die künftige Tarifstruktur soll nun einen direkten Leistungsbezug aufweisen. Hierzu müssen die für die Patientinnen und Patienten in der Rehabilitation erbrachten Leistungen in der Prozedurenklassifikation CHOP abgebildet sein und von den Leistungserbringern entsprechend erfasst werden.

\section{Fachgesellschaften definieren Basis- und Zusatzleistungen}

Inzwischen konnte auf dem Weg zu einer leistungsbezogenen und CHOP-Kode-basierten Tarifstruktur in der stationären Rehabilitation eine weitere Etappe erfolgreich zurückgelegt werden. Dazu hat die Ärzteschaft einen wesentlichen Beitrag geleistet. In ihrer über zweijährigen Tätigkeit haben die FMHExperten und die betroffenen Fachgesellschaften die Basis- und Zusatzleistungen der Rehabilitation definiert. ${ }^{1}$ Basisleistungen sind jene Leistungen, die der Patient oder die Patientin mindestens erhalten soll, wie z.B. eine Eintrittsabklärung, eine Austrittsplanung oder regelmässige Visiten. Die Definition von Basisleistungen mit Mindestanforderungen entspricht dem Anliegen der Fachgesellschaften, die Qualität in der Rehabilitation zu gewährleisten. Die Spezifizierung von Zusatzleistungen wiederum dient dazu, besonders komplexe und kostenaufwendige Rehabilitationsfälle möglichst leistungsgerecht zu vergüten. Zusatzleistungen kommen nur im Falle eines ausgewiesenen Bedarfs zur Anwendung.
Die aufwendigen Arbeiten der FMH und ihrer Fachgesellschaften haben sich gelohnt, denn sie sind in die CHOP-Version 2019 eingeflossen. Dies hat eine Kodebasierte Leistungserfassung überhaupt erst möglich gemacht. Die Fachgesellschaften konnten ihre Anliegen bestmöglich einbringen, obwohl Kompromisse gegenüber den Partnern der SwissDRG AG erforderlich waren.

Im Anschluss an die Veröffentlichung der definierten Basis- und Zusatzleistungen in der CHOP 2019 mussten nur wenige Anpassungen vorgenommen werden. Diese hat das Bundesamt für Statistik (BFS) kürzlich in seinem Rundschreiben publiziert. ${ }^{2}$ Erwähnenswert ist dabei insbesondere die Anpassung des CHOP-Kodes «BB.1 Zusatzaufwand in der Rehabilitation»: Neu wird hier auf die Angabe spezifischer Facharzttitel als Voraussetzung für die Kodierung verzichtet. Leserfreundlich

Zentral wird sein, dass die Kliniken die neuen CHOP-Kodes 2019 erfassen, denn diese werden die Grundlage für die Einführungsversion ST Reha bilden.

aufbereitet und auf dem neusten Stand sind die Basisund Zusatzleistungen der CHOP 2019 inkl. dieser Änderungen auch auf der FMH-Website zu finden. ${ }^{3}$

\section{Personelle und infrastrukturelle Minimalanforderungen erarbeitet}

Als weiteres zentrales Puzzleteil haben die FMH und ihre Fachgesellschaften einen Vorschlag zur Spezifizierung der personellen und infrastrukturellen Minimalanforderungen erarbeitet und diesen den Partnern zur Diskussion vorgelegt. Es handelt sich dabei um 
4 Neurologische, psychosomatische, pulmonale, kardiale, muskuloskelettale, internistisch/ onkologische, pädiatrische und geriatrische Rehabilitation.

5 www.fmh.ch $\rightarrow$ Stationäre Tarife $\rightarrow$ Rehabilitation ST Reha

6 myfmh.fmh.ch

7 Zusatz zum medizinischen Kodierungshandbuch, Version 2019: Kodierrichtlinien für die Rehabilitation, www.bfs. admin.ch $\rightarrow$ Statistiken finden $\rightarrow$ Gesundheit $\rightarrow$ Medizinische Kodierung und Klassifikationen $\rightarrow$ Instrumente zur medizinischen Kodierung

Korrespondenz:

FMH

Abteilung Stationäre Versorgung und Tarife

Baslerstrasse 47

$\mathrm{CH}-4600$ Olten

Tel. 0313591111

Fax 0313591112

tarife.spital[at]fmh.ch
Voraussetzungen, welche die Kliniken für die Erbringung der Basisleistungen je nach Rehabilitationsart ${ }^{4}$ erfüllen sollen. Nach intensiven Verhandlungsrunden konnten sich die Partner der SwissDRG AG schliesslich auf eine Kompromissversion einigen, die als integraler Bestandteil der CHOP-Klassifikation und in Form von Referenzdokumenten für jede der acht Rehabilitationsarten auf der Website $^{5}$ der FMH veröffentlicht wurde. Die Referenzdokumente spezifizieren in erster Linie personelle und infrastrukturelle Anforderungen, beispielsweise hinsichtlich der Leitung des Behandlungsteams, der ärztlichen und pflegerischen Präsenz oder der verfügbaren Diagnostik (vgl. Übersicht 1). Um den administrativen Aufwand zu begrenzen, sind die Partner der SwissDRG AG übereingekommen, dass diese Kriterien jeweils von der leistungserbringenden Einrichtung oder Abteilung zu erfüllen sind, nicht aber für jeden Einzelfall separat dokumentiert werden müssen.

Übersicht 1: Kriterien personeller und infrastruktureller Minimalanforderungen

- Definition der spezifischen Reha-Art

- Indikation für die Durchführung der spezifischen Reha-Art

- Ziel der spezifischen Reha-Art

- Leiter des Behandlungsteams (z.B. fachärztliche Voraussetzungen und Anstellungsgrad)

- Ärztliche und pflegerische Präsenz (z.B. zeitliche Verfügbarkeiten vor Ort)

- Behandlungsteam (z.B. Beschreibung der beteiligten Professionen aus den Bereichen Medizin, Pflege, Therapie)

- Infrastruktur (z.B. spezifische Diagnostik und Rollstuhlgängigkeit)

- Basic Life Support (z.B. Verfügbarkeit an 24/24 Stunden und 7/7Tagen)

\section{FMH-Dokumentationsmuster zur Unterstützung der Leistungserfassung}

Entscheidend wird sein, dass die Kliniken die neuen CHOP-Kodes der Basis- und Zusatzleistungen für die Rehabilitation erheben. Dies deshalb, weil die SwissDRG AG die Einführungsversion ST Reha anhand der Daten 2019 kalkulieren wird. Nur so wird die Einführung wie geplant per 1. Januar 2022 möglich sein. Zur Unterstützung der Leistungserfassung in den Kliniken hat die FMH für die Basis- und Zusatzleistungen in der Rehabilitation Dokumentationsmuster erstellt, die allen FMH-Mitgliedern zur Verfügung stehen. ${ }^{6}$ Als eine Art Checkliste wurden die Informationen über die erbrachten Leistungen möglichst einfach und CHOP-konform zusammengefasst und kön- nen an die klinikspezifischen Bedürfnisse angepasst werden. Als Zusatz zum medizinischen Kodierungshandbuch wurden mit Unterstützung der FMH für Kliniken zudem Kodierrichtlinien für die Rehabilitation ausgearbeitet und schliesslich vom BFS veröffentlicht.

\section{Detaillierte Austrittsberichte erfüllen mehrere Zwecke}

In den Austrittsberichten der Rehabilitation sind relevante Informationen wie Haupt- und Nebendiagnosen Angaben zu durchgeführten Therapien sowie Empfehlungen für weitere Behandlungen enthalten. Ergänzt werden diese Angaben um zusätzliche Aufwände wie beispielsweise eine erfolgte Isolation mit Angabe der Isolierungstage, extern durchgeführte diagnostische Massnahmen oder Therapien (z.B. Dialyse). Dabei sind solch detaillierte Informationen nicht nur für die weiterbehandelnden Ärztinnen und Ärzte notwendig, sondern auch für die Kodierung der Reha-Fälle, bei Rückfragen seitens der Krankenversicherer oder bei der Kodierrevision.

\section{Datengrundlage schrittweise verbessern}

Dank dem Engagement der Fachgesellschaften und der Kompromissbereitschaft der Partner der SwissDRG AG liegen heute CHOP-Kodes für die Basis- und Zusatzleistungen inkl. der personellen und infrastrukturellen Minimalanforderungen vor. Wichtig ist nun, dass die Kliniken ihre Kosten- und Leistungsdaten 2019 als Grundlage für die Einführungsversion ST Reha gut erfassen. Die Erfahrungen, welche die Kliniken mit der Leistungsdokumentation und -kodierung in den ersten Monaten des laufenden Jahres machen, können zudem für die Systemweiterentwicklung hilfreich sein. So werden beispielsweise die Fachgesellschaften mit Unterstützung der FMH-Experten der Abteilung Stationäre Versorgung und Tarife prüfen, welche weiteren Zusatz-

Die FMH-Experten unterstützen die Fachgesellschaften bei Anträgen zur sachgerechten Abbildung rehabilitativer Leistungen in der CHOP.

leistungen besonders aufwendig sind, und entsprechend Anträge für künftige CHOP-Versionen stellen. Auf dem Weg zu einer leistungsgerechten Tarifstruktur in der stationären Rehabilitation bleibt noch einiges zu tun, aber ein wichtiger Grundstein ist nun gelegt. 